\title{
Movilidad femenina en Santiago de Chile: reproducción de inequidades en la metrópolis, el barrio y el espacio público'
}

\author{
Mobilidade feminina em Santiago do Chile: reprodução das inequidades na \\ metrópole, no bairro e no espaço público
}
Women's mobility in Santiago, Chile: Reproduction of inequalities in the metropolis, neighbourhood, and public space

Cristhian Figueroa Martínez ${ }^{[0]}$ Natan Waintrub Santibáñez ${ }^{[b]}$

\footnotetext{
[a] Pontificia Universidad Católica de Chile, Facultad de Arquitectura, Diseño y Estudios Urbanos, Escuela de Arquitectura, Santiago, Chile

[b] Pontificia Universidad Católica de Chile, Facultad de Ingeniería, Escuela de Ingeniería, Santiago, Chile
}

\section{Resumen}

La construcción social que asocia a las mujeres con la mantención y cuidado del hogar las muestra como un grupo con baja disponibilidad de tiempo y con limitadas capacidades de desplazamiento urbano. La limitación se traduce en una movilidad diferenciada, acotada y vulnerable a fenómenos tan diversos como su lugar de residencia y la calidad del espacio público. En el caso de Santiago de Chile, existen diferencias considerables en la movilidad según género, edad y estrato socioeconómico, destacándose una mayor fragilidad en grupos de escasos recursos que habitan en barrios producidos por el Estado durante la segunda mitad del siglo pasado. Mediante indicadores cuantitativos y herramientas cualitativas, el presente texto tiene por propósito caracterizar la movilidad femenina en Santiago en tres escalas: metropolitana, barrial y local. La evidencia muestra que existen disparidades en los todos los niveles. En la escala metropolitana las mujeres de ingresos altos exhiben un elevado número de viajes, mayoritariamente motorizados, mientras que en los grupos de escasos recursos dominan los desplazamientos pedestres. En una escala menor, en los barrios, los traslados peatonales se encuentran fuertemente afectados por factores sociales y ambientales como, por ejemplo, la percepción de seguridad.

Palabras Clave: Movilidad. Mujeres. Transporte.

Resumo

A construção social que relaciona as mulheres com a manutenção e a supervivência do lar as mostra como um grupo com baixa disponibilidade de tempo e com limitadas capacidades de desplazamento urbano. A limitação se traduz em uma mobilidade diferenciada, acotada e vulnerável aos fenômenos tão diversos como

\footnotetext{
1 Parte de este trabajo está basado en la tesis para optar al grado de Magíster en Proyecto Urbano de Cristhian Figueroa Mar t í n e z "Recalificación de la estructura del espacio público según la movilidad cotidiana de las mujeres de la población Santa Julia de Macul”, 2011, Pontificia Universidad Católica de Chile. Profesor Guía Rosanna Forray Claps.
}

CFM é Arquitecto, magíster en Proyecto, e-mail: coiguer@uc.cl

NWS é Licenciado en Ciencias de la Ingeniería, magister(c) en Ciencias de la Ingeniería, Mención Transporte y Logística, e-mail: nwaintrub@uc.cl 
o seu lugar de residência e a qualidade do espaço público. No caso de Santiago do Chile, existem diferenças consideráveis na mobilidade segundo gênero, idade e estrato socioeconômico, destacando uma maior fragilidade em grupos de escassos recursos que vivem em bairros produzidos pelo Estado durante a metade do século passado. Por meio de indicadores quantitativos e ferramentas qualitativas, o texto seguinte tem como propósito caraterizar a mobilidade feminina em Santiago do Chile em três níveis: metropolitano, de bairro e local. A evidência mostra que existem disparidades em todos os níveis; em escala metropolitana as mulheres de baixo ingresso exibem um elevado número de viagem, maioritariamente motorizados, enquanto que em grupos de escassos recursos predomina os deslocamentos a pé. Em uma escala menor, nos bairros, a circulação de pedestres se encontra fortemente afetada por fatores sociais e ambientais, como por exemplo pela percepção de segurança.

Palavras-chave: Mobilidade. Mulheres. Transporte.

\section{Abstract}

The social construction that links women to the support and survival of households characterizes them as a group with low amount of available time and limited capacities to perform urban trips. This limitation is translated into a different mobility, which is enclosed and vulnerable to diverse phenomena such as residential location and quality of public space. In the case of Santiago, Chile, there are considerable mobility differences depending on gender, age and income; emphasizing the higher fragility of low income groups that inhabit the neighborhoods created by the State during the second half of the past century. Through quantitative indicators and qualitative tools, the present paper aims to characterize three scales of women's mobility in Santiago: metropolitan, neighborhood, and local. The evidence shows disparities at all the levels: in the metropolitan scale, high income women perform a high number of trips, and most of them are motorized; on the other hand, pedestrian trips are predominant in the low income groups. In a smaller scale, in the neighborhoods, pedestrian trips are strongly affected by social and environmental factors such as security perception.

Keywords: Mobility. Women. Transport.

\section{Introducción}

Diversos autores describen la movilidad como un fenómeno mediante el cual las personas satisfacen sus necesidades de conexión (Ascher, 2005; Módenes, 2007; Kaufmann, 2001). Por el lado de la economía, la movilidad es un bien derivado consumido para obtener otro (Ortúzar \& Willumsen, 2011). Debido a esta particularidad, la movilidad es un bien que genera des-utilidad, siendo deseable no consumirlo. Consecuentemente, las personas siempre buscarán reducir todos sus costos asociados; temporales, monetarios, transbordos, confort, entre otros.

Desde un punto de vista territorial, Hercé (2009) indica que la movilidad expresa las oportunidades de conexión física o virtual ofrecida por las ciudades, y está influenciada tanto por factores socioeconómicos, tales como las capacidades físicas y económicas que cada persona tiene, dependiendo de los recursos familiares de donde proviene, como por constructos sociales como el género.

Rainero (2001) considera que las mujeres conforman uno de los grupos con movilidades más complejas debido a una fuerte construcción social que las asocia con la supervivencia económica, funcional y social del hogar. La mujer es la encargada de asegurar la superviviencia de su grupo familiar en el tiempo y el espacio, limitando su tiempo disponible y, disminuyendo con ello, sus capacidades de desplazamiento.

Esta restricción temporal es más aguda en sectores precarios de ciudades desiguales como las latinoamericanas, en donde a la mantención del hogar, se le suman otras dificultades: ausencia de redes sociales de apoyo, de transporte público confiable y de calidad, y la percepción de inseguridad. 
La movilidad en estos territorios es vulnerable y se compone por desplazamientos amenazados, en riesgo de desaparecer (Gutiérrez, 2007).

Santiago de Chile, no exenta de las problemáticas de la región, evidencia una fuerte disparidad en el acceso a las oportunidades que ofrece la ciudad. Esta desigualdad se refleja en la directa relación que existe entre movilidad femenina y pobreza: cuanto menor es la movilidad de la mujer, menores son los indicadores socio-económicos, tanto los propios como los del grupo familiar al que pertenece (Valenzuela, 2003; Rainero, 2001).

El objetivo del presente texto es caracterizar la movilidad de las mujeres de bajos recursos que habitan Santiago. Se analizará detalladamente tanto la movilidad de las mujeres de forma global en la metrópoli como sus diferencias con respecto a la movilidad masculina. Y, a escala de barrio, se caracterizará la relación entre percepción de seguridad y espacio público.

En términos metodológicos, el texto presenta una mixtura de herramientas cuantitativas y cualitativas. En la escala metropolitana se utilizaron los datos obtenidos por la Encuesta Origen Destino (EOD) de los años 1991 y 2006 (SECTRA, 1991, $2006)^{2}$, analizando el número de viajes por persona, partición modal, motivo de viaje y tiempos de viaje por edad e ingreso económico. Para comprender las características generales de la movilidad del género femenino, se referencian en la escala metropolitana algunos datos relevantes sobre el género masculino.

A escala barrial, mediante registro y trabajo de campo, se levantaron las redes de movimiento peatonal femenino de Santa Julia de Macul, la población de vivienda social más cercana al centro financiero ${ }^{3}$. Posteriormente, en una segunda etapa, se encuestó a las mujeres que circulan dentro de dicha

\footnotetext{
2 Corresponde a la última base de datos de movilidad de Santiago de Chile que no incluye los efectos que Transantiago (implementado en 2007) podría tener sobre algunos indicadores como, por ejemplo, la partición modal.

3 El registro de campo se realizó mediante conteos de 10 minutos en todo el territorio de la población durante los horarios en que, de acuerdo a la literatura, circula un mayor número de mujeres por el espacio público (entre 8:00 y 14:00 horas, en Daniels \& Warne (1993) y SECTRA (2006). Asimismo, el registro se realizó entre los meses de abril y mayo, y durante los días martes, miércoles y jueves que, según las encuestas de movilidad de Santiago de Chile, registran comportamientos similares (SECTRA, 2006).
}

población, con el fin de indagar en los motivos por los que eligieron unas rutas por sobre otras ${ }^{4}$.

Con el fin de vincular ambas escalas e introducir las problemáticas observadas en el apartado cualitativo, se hizo un estudio cuantitativo de Santa Julia y de otras tres zonas vulnerables de Santiago ${ }^{5}$ (Juanita Aguirre, Las Turbinas y Santo Tomás). Para estas últimas se registraron el número de viajes por persona, la partición modal y la proporción de viajes realizados fuera de la población según las EOD de los años 1991 y 2006.

\section{Movilidad femenina vulnerable}

La literatura considera a la mujer como un grupo históricamente relegado de la esfera pública y la toma de decisiones; es un grupo fuertemente ligado a la mantención del hogar (Izard, 1985; Valcárcel \& Quiroz, 2008). La exclusión de lo público disminuye en parte con las diversas luchas de reivindicación de derechos realizadas durante la segunda mitad del siglo XX (Perrot, 1997). No ajena a esta tendencia, en la temática urbana emergieron movimientos de similares características que reconocieron a la mujer como un ser apartado de la sociedad urbana y de sus dinámicas sociales.

El movimiento Feminista, desarrollado en Estados Unidos, argumenta que la dispersión urbana, la monofuncionalidad y la baja densidad (sprawl) enclavaron a la mujer al hogar (Hayden, 1981; Rosenbloom, 2004). Las distancias fueron aumentando y, como consecuencia, el automóvil surgió como la única solución para movilizarse y realizar las actividades cotidianas. Quien no tiene capacidades físicas y recursos para obtener y conducir un automóvil queda inmovilizado e incapacitado para acceder a las oportunidades que las ciudades ofrecen.

\footnotetext{
4 Se encuestaron a 200 mujeres que circulan por el espacio público de la población. Parte de la encuesta poseía preguntas abiertas que abordaban las características de los espacios públicos prohibidos a la circulación.

5 La elección de estas tres zonas (Juanita Aguirre, Las Turbinas y Santo Tomás) obedece a criterios de homogeneidad social y número de habitantes. La zona de Santa Julia corresponde al estudio de caso de la tesis en que se basa parcialmente el presente texto.
} 
En este contexto, la mujer se queda a cargo del cuidado del hogar y de y de todos los viajes que ello implica (compras, diligencias, entre otros). Además, como el automóvil automáticamente inmoviliza a niños, adolescentes, ancianos e incapacitados físicos, imposibilitados legal o técnicamente para conducir, la mujer debe actuar como chófer o compañía, trasladándolos de un recinto a otro para que puedan realizar sus actividades cotidianas (Law, 1996; Handy, 2004; Markovich \& Hendler, 2006).

Aunque la descripción feminista del rol de la mujer fue desarrollada en una sociedad centrada en el automóvil, muy diferente a las de los países en desarrollo, como los latinoamericanos, dicha descripción ejemplifica claramente las características de la movilidad femenina: múltiples desplazamientos relacionados con el cuidado del hogar y la mantención de terceras personas. Todos estos traslados impactan en la vida cotidiana de las mujeres, disminuyendo su tiempo disponible y aumentando dramáticamente su valor y la forma de disponer de él (Alcaíno \& Gutiérrez, 2009).

Siguiendo este argumento, si se comprende que cada desplazamiento por la ciudad es el resultado de una ecuación que sopesa el costo monetario y temporal del desplazamiento (McFadden, 1974) con el del valor del bien a obtener (Daniels \& Warnes, 1993; Perulli, 1995), se entiende que por la alta valoración que las mujeres asignan al tiempo se produzca en ellas una mayor tendencia a la inmovilidad. Sólo se desplazarán si el bien les genera una alta utilidad, sin que llegue a comprometer su hogar y sus integrantes. Cuando el costo es demasiado alto, las mujeres abandonarán el trabajo para asegurar la supervivencia del hogar (Jirón, 2007).

Como el valor de los bienes a obtener tiende a la estabilidad y obedece las normas de mercado, las mujeres modifican la ecuación alterando el costo de los desplazamientos. Para ello, articulan estrategias, de tal forma que en un solo viaje se sumen varios propósitos (trip-chaining, Primerano et al., 2008) o bien disminuyen los tiempos de traslado (Figueroa \& Cortés, 2013), aumentando la velocidad, acortando las distancias del trayecto o modificando los destinos. Pero por otro lado, esta ecuación de costos también se realiza en los modos de transporte que utilizan las mujeres, tendiendo a hacer el trade-off entre costo del modo versus tiempo de viaje, acceso y espera.
La primera posibilidad indica que debe existir un medio de transporte que reduzca el tiempo (por ejemplo el automóvil) o una infraestructura que aumente la velocidad y la accesibilidad a un lugar determinado (autopistas, trenes, entre otros). La segunda opción implica restringir el radio de traslado, limitando los desplazamientos a áreas geográficas acotadas cuyo centro sea el lugar de residencia. El problema de esta segunda alternativa radica en que el área acotada, el área sobre el que se restringe el desplazamiento, no necesariamente provee la solución a todas las necesidades vitales 0 , si las provee, no necesariamente son de calidad. Por ejemplo, en el caso chileno, Thieme (2010) apunta que las familias escogen escuelas primarias cercanas sin importarles la calidad de su enseñanza.

En el contexto Latinoamericano, con ciudades altamente segregadas y con severos problemas de accesibilidad, la limitación del radio de acción de las mujeres trae consigo una fuerte tendencia a realizar desplazamientos pedestres (Alcaíno et al., 2009). La tendencia a la caminata y al cuidado del hogar aumentan, en el género femenino, a medida que disminuye su nivel socio-económico; las mujeres con menos recursos económicos son las que proporcionalmente realizan más traslados peatonales por la ciudad, ya sea por la falta de automóvil, de transporte público eficiente y confiable, o por la distancia geográfica entre su domicilio y los lugares de trabajo, entre otros. Así, por añadidura, Banister (1994) indica que los grupos de escasos recursos se mueven menos y utilizan medios de transporte local como autobuses, generando una paradoja en donde las grandes infraestructuras, y los recursos e inversiones que son demandados son regresivos, beneficiando desproporcionadamente más a las clases medias y altas.

La gran cantidad de desplazamientos pedestres que realizan las mujeres trae como consecuencia una constante y fuerte relación con sus barrios de habitación y las características del espacio público por el cual deben circular. Así, Aguirre (1992) apunta que debido a esta constante y fuerte relación, las mujeres son actores claves para la elaboración de políticas públicas orientadas a la mejora de áreas precarias; son ellas las que verdaderamente conocen las necesidades de sus barrios.

Sin embargo, el excluir a las mujeres de la esfera pública, trae como consecuencia una mayor fragilidad 


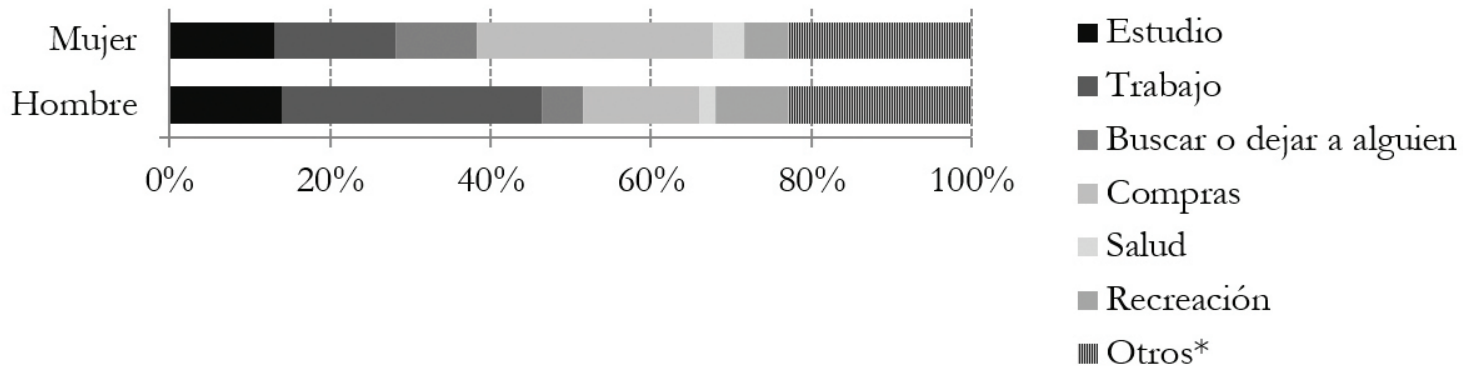

Figura 1 - Motivos de viaje según género

Fuente: SECTRA (2006).

Nota: *motivos de viaje que representan menos del $3 \%$ en ambos grupos y cuya diferencia porcentual entre hombres y mujeres no supera el $2 \%$.

y desconfianza de los espacios públicos por los que perambulan, impidiendo todavía más su movilidad (Loukaitou-Sideris, 2004). De esta manera, como indica Rainero (2006) y Rodigou (2009), la percepción de inseguridad derivada de las condiciones del espacio público (deterioro, nivel de oscuridad, presencia de basura) y de los símbolos presentes en él (grafitis, marcas de territorio de pandillas, entre otros) modifica el comportamiento y tiende a restringir las posibilidades de desplazamiento. La percepción es capaz, en casos extremos, de reprimir traslados (Rainero, 2006).

Por este motivo, al que se añaden las características intrínsecas del género femenino expuestas con anterioridad, Gutiérrez (2007) apunta que las mujeres de escasos recursos desarrollan una movilidad "vulnerable", compuesta por traslados en riesgo de no ser realizados. Esta vulnerabilidad es uno de los factores que influyen en la tendencia al empobrecimiento del género femenino (Valenzuela, 2003; Rainero, 2001).

\section{Movilidad femenina en Santiago de Chile}

A escala metropolitana, Santiago de Chile posee poco más de 6 millones de habitantes y genera más de 16 millones de viajes en un día hábil promedio (SECTRA, 2006). Más en detalle, la ciudad muestra 2,80 viajes por persona que se dividen en 2,77 para hombres y 2,83 para mujeres. De acuerdo a este primer indicador las mujeres tenderían a realizar un número mayor de viajes por la ciudad; sin embargo, si se agrega el tiempo de viaje, se desprende que los viajes de las mujeres utilizan un 13,8\% menos de tiempo que el de los hombres (SECTRA, 2006).
Los viajes de las mujeres son comparativamente más cortos que los que realizan los hombres. La tendencia incluso parece aumentar en favor de los hombres, pues a inicios de la década de 1990 los desplazamientos femeninos eran un 12,9\% más cortos que los desplegados por los hombres (SECTRA, 1991).

En relación a las motivaciones de los desplazamientos en ambos géneros, el motivo "estudio" agrupa un porcentaje similar de viajes. El trabajo representa más del 35\% de los viajes masculinos, mientras que en los femeninos son sólo de un $15 \%$. Contrastamos que las compras (29\%), la salud (4\%) y buscar y dejar a alguien (10\%) explican casi la mitad de los viajes realizados por mujeres y sólo una quinta parte de los traslados de los hombres (14,5\% en compras, $1,9 \%$ en salud y $5 \%$ en buscar o dejar a alguien). Una diferencia interesante aparece en el ítem "recreación" que concentra un 9\% de los viajes en hombres y solo un 5\% en mujeres (Figura 1).

Sin embargo, la EOD anterior muestra que las diferencias, salvo en los motivos de trabajo y estudios, ha tendido a acentuarse con el tiempo. De esta manera, hacia 1991 los hombres realizaban el 50\% de sus viajes al trabajo y las mujeres un $26 \%$; mientras que los estudios representaban un $27 \%$ para los hombres y un $29 \%$ para las mujeres. En contraste, los viajes para realizar diligencias ${ }^{6}$ muestran un diferencial de $10 \%$ a favor de las mujeres (18\%), mientras que las compras motivan el $10 \%$ de los viajes en mujeres y tan sólo el $4 \%$ en hombres (SECTRA, 1991).

6 Viajes realizados por trabajo para realizar pagos de cuentas, trámites, citas en centros de salud, etc. 


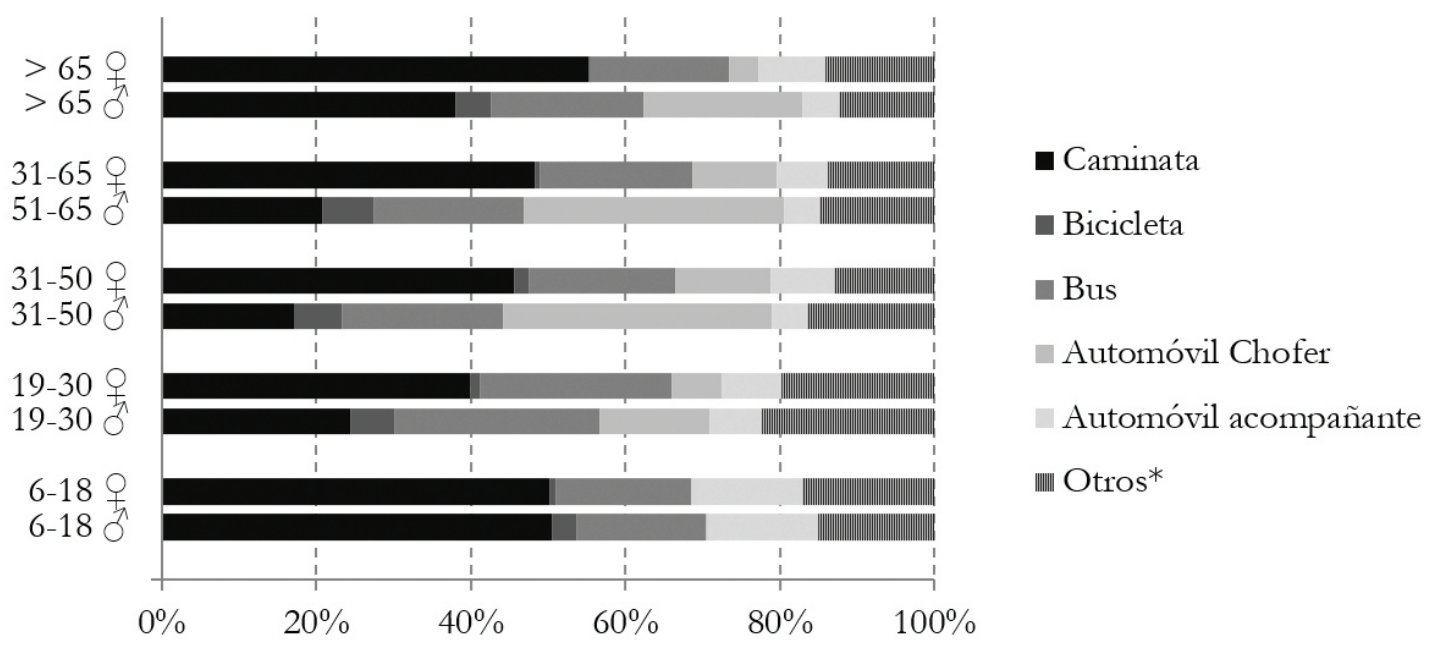

Figura 2 - Partición modal según género y edad Fuente: SECTRA (2006).

Nota: * ${ }^{\star}$ corresponde a todos aquellos medios de transporte que no representan más del $2 \%$ de los desplazamientos de hombres y mujeres.

Las diferencias motivacionales de los desplazamientos en hombres y mujeres muestran los distintos roles con los que tradicionalmente se les asocia. Por un lado, entre los hombres, con el rol de proveedor, dominan los desplazamientos de trabajo; mientras que entre las mujeres, con el rol asociado al hogar y sus labores cotidianas, las actividades asociadas a la mantención de terceras personas y las compras y las actividades asociadas a la mantención de terceras personas agrupan la mayor parte de los desplazamientos (salud y buscar o dejar a alguien).

En cuanto a partición modal, las mujeres que habitan Santiago tienden a caminar más que los hombres, con porcentajes de 55,32\% y 39,57\%, respectivamente. Asimismo, las mujeres tienden a usar el taxi y el taxi colectivo ${ }^{7}$ más que los hombres, pero estos viajes representan una pequeña fracción de la partición modal total $(1,45 \%$ y $2,96 \%$ de los viajes son en taxi y taxi colectivo, respectivamente). Nuevamente la desigualdad parece reforzarse con el tiempo, ya que a principios de la década de 1990 la caminata representaba sólo un $25,51 \%$ para las mujeres y un 17,90\% para los hombres (SECTRA, 1991).

Las desigualdades entre géneros son aún más evidentes cuando se considera la evolución de la partición modal durante la vida de las personas. Aunque no se observan diferencias relevantes en los

\footnotetext{
7 Taxis con recorrido fijo compartidos por un máximo de cuatro
} pasajeros. medios utilizados por niños y jóvenes, salvo el uso de la bicicleta, la partición modal en este grupo tiende a ser similar entre ambos géneros. Sin embargo, los tramos de edad comprendidos entre 19-30 y 31-65 años exhiben importantes asimetrías en el uso de los distintos modos de transporte (Figura 2).

El transporte público y el automóvil particular tienden a mantenerse constantes y equitativos tanto para hombres como para mujeres en los distintos tramos etarios, aunque no sucede lo mismo con la caminata y el automóvil. La caminata para las mujeres entre 31 y 50 años congrega más del $40 \%$ de los desplazamientos, en tanto que para los hombres la cifra se reduce a menos del $30 \%$. Por otro lado, el automóvil es utilizado por más del $30 \%$ de los hombres de entre 31 y 50 años, en tanto que para las mujeres sólo llega al 12\% del número total de viajes que realizan (Figura 2).

En proporción, los hombres duplican el uso del automóvil en todos los tramos de edad. Las mujeres muestran un fenómeno similar con la caminata. Esto deja al descubierto la diferencia de movilización que poseen ambos géneros: el predominio de los automóviles entre los hombres indica que tienen capacidad para realizar viajes de largo alcance, mientras en las mujeres sucede lo contrario, al predominar la caminata por encima de cualquier otro medio de locomoción.

La partición modal y la edad también evidencian la edad productiva y reproductiva de ambos géneros, y muestran la ausencia de diferencias en la infancia 


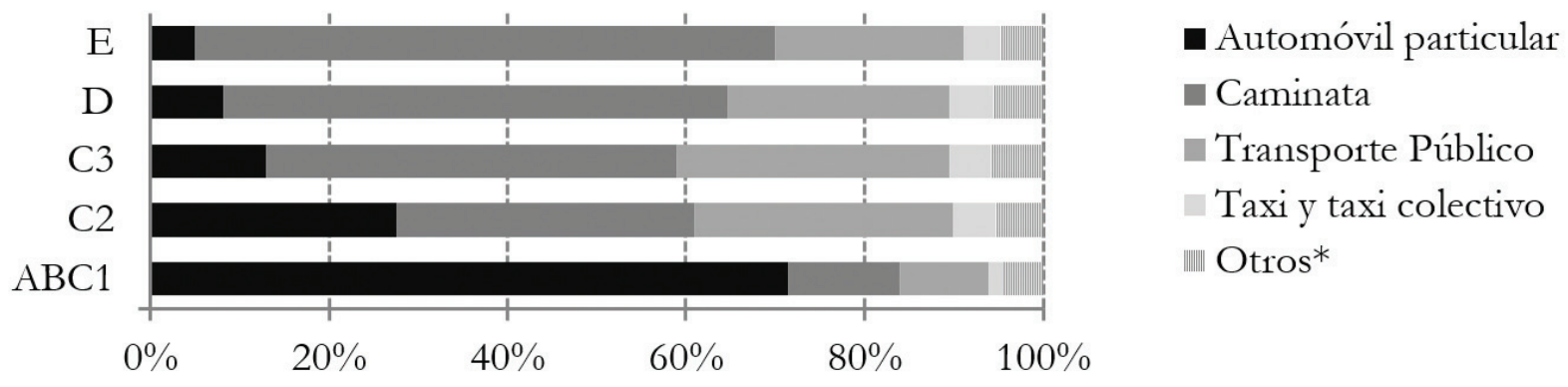

Figura 3 - Partición modal de mujeres según estratos socio-económicos Fuente: SECTRA (2006).

Nota: ${ }^{*}$ motivos de viaje que representan menos del $3 \%$.

y su atenuación en la tercera edad (mayores de 65). Los adultos mayores son el único rango de edad en donde la caminata explica más de un tercio de los desplazamientos que realizan diariamente los hombres. Entre las mujeres, las mayores diferencias en el caso de Santiago están dadas por el estrato socio-económico al que pertenecen sus familias ${ }^{8}$. Como era de esperar, los sectores más adinerados son los que utilizan el automóvil con una proporción de 71,45\% (Figura 3). En los estratos bajos predomina la caminata, llegando a concentrar más de la mitad de los desplazamientos femeninos en los dos grupos de más bajos ingresos (D y E).

Resalta el uso casi por igual del bus y taxi-bus por los C2, C3, D y E. De ello se deduce que el transporte público en la ciudad esté disociado de los grupos de ingreso alto y vinculado a los estratos medio y bajo. En este contexto, las mujeres de estratos altos tienden a movilizarse más, ya que, a diferencia del transporte público que, por sus características intrínsecas, posee rutas fijas, el automóvil les permite adecuar recorridos y horarios a sus necesidades. La flexibilidad queda expuesta al observar que los estratos $\mathrm{AB}, \mathrm{C} 1, \mathrm{C} 2$ y C3 presentan más de 3 viajes por persona, disminuyendo a 2,71 para estratos $\mathrm{D} y$ a 1,94 para el estrato E.

Además, el automóvil permite acceder a territorios que no necesariamente están conectados con

8 Se utilizó la estratificación definida según SECTRA (2006), actualizado según el IPC (índice de precios al consumidor) para la EOD 2006, donde el ingreso mensual del estrato bajo (D y E) corresponde a un ingreso inferior a US 697 (CHL \$383.352), medio (C2 y C3) entre US 697 (CLP \$383.354) hasta US 1590 (CLP \$874.394) y alto (AB y C1) superior a US 1590 (CLP 874.395). transporte público, encadenar actividades en un solo recorrido y optimizar el uso de su tiempo (Valiquette \& Morency, 2010). No es de extrañar que, dada esta flexibilidad dentro del grupo de ingresos altos, el trabajo agrupe más de un 19,82\% de los motivos de viaje, pues sus recursos de movilidad les permite optimizar el tiempo y acceder a otro tipo de oportunidades, inaccesibles para aquellos grupos de menores recursos y dependientes del sistema de transporte público.

Al respecto, Markovich \& Hendler (2006) indican que, de una manera perversa, disponer de un automóvil representa una forma "eficaz" de salir de la pobreza en contextos donde el transporte público no es ni fiable ni eficiente y/o donde la forma urbana no permite la existencia de modos de transporte masivo (suburbios densos y distantes del centro de la ciudad). Tener automóvil en estos contextos es sinónimo de acceso a las oportunidades de la ciudad.

En el caso de Santiago, debido a agresivas políticas de vivienda económica y eficiencia económica, se expulsó la pobreza a los márgenes de la ciudad (Hidalgo, 2005; Sabatini, 2000). A una periferia que, aunque tenga transporte público, carece de oportunidades laborales y servicios de calidad. Por el contrario, la riqueza se concentra en un área triangular que se extiende desde el centro histórico hacia el oriente (Figura 4). Dentro de esta misma área se sitúa el centro financiero de la ciudad concentra más del $90 \%$ de los metros cuadrados de oficinas de la metrópoli y sólo dos comunas (Santiago y Providencia) atraen más de la mitad de los desplazamientos de trabajo durante las horas punta, dificultando aún más los desplazamientos de los grupos desfavorecidos que habitan en una 

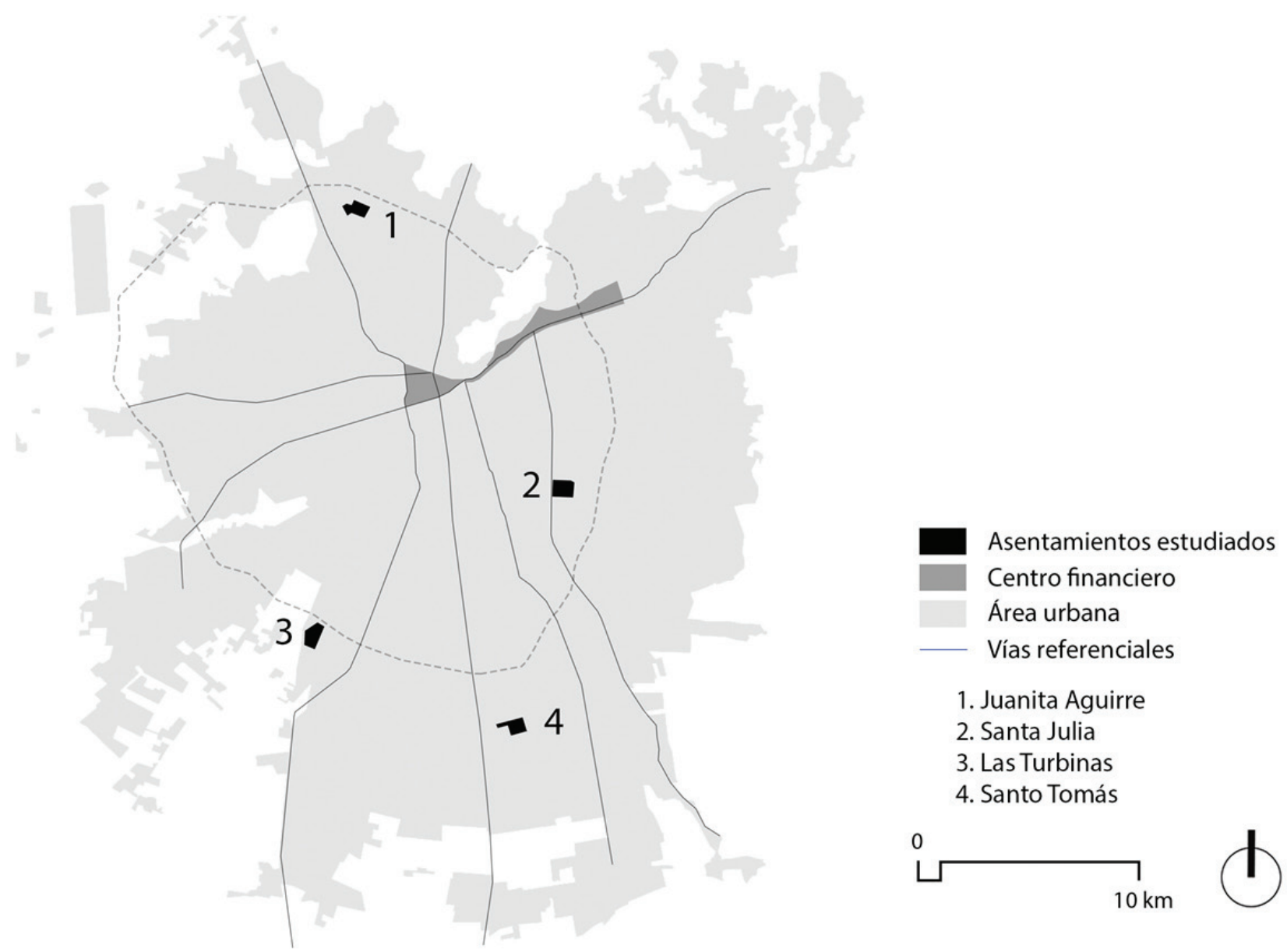

Figura 4 - Estructura de Santiago y localización de los casos

Fuente: elaboración propia.

periferia distante y densamente poblada (Tokman, 2006).

La articulación de esta periferia de bajos recursos se llevó a cabo mediante una sucesión de nuevos asentamientos que aumentaron rápidamente el tamaño de la ciudad durante la segunda mitad del siglo $\mathrm{XX}$. Estos asentamientos inicialmente fueron barrios equipados y -en teoría- autosuficientes (hasta 1973), aunque luego fueron transformados en territorios residenciales monofuncionales, sin equipamientos ni servicios (a partir de 1973, Sugranyes, 2005). Por estos motivos, las desigualdades que anteriormente se observaron a nivel metropolitano reaparecerán bajo nuevas formas a nivel de barrio.

Cuatro casos seleccionados -Santa Julia, Juanita Aguirre, Santo Tomás y Las Turbinas ${ }^{9}$ - muestran

9 Los dos primeros casos (Santa Julia y Juanita Aguirre) fueron originados por la política de vivienda llamada "Operación que la caminata es el medio más utilizado por las mujeres. En los sectores de Santa Julia y Santo Tomás la caminata congrega más del $63 \%$ de los viajes, mientras que en Las Turbinas y Juanita Aguirre corresponde a un $47,60 \%$ y $36,43 \%$ respectivamente (Figura 5). Por otro lado, el sector Juanita Aguirre es el único en donde este modo sobrepasa el 10\% de los traslados femeninos $(16,2 \%)$.

Las explicaciones tras la alta participación de la caminata en Santo Tomás y Santa Julia pueden ser diversas pero resulta evidente al menos una

Sitio" (1965-1970) que localizó a los grupos carentes en sitios cercanos a los asentamientos irregulares originales, en barrios con equipamientos interior (Hogar de Cristo, 1973). Los sectores de Santo Tomás y Las Turbinas fueron producto de un cambio en la política que buscó externalizar a manos privadas los costos de producción de vivienda social (1985-2002), trasladando las grandes poblaciones ubicadas en el centro hacia barrios periféricos exclusivamente residenciales (Sugraynes, 2005). 
situación; diversidad de actividades en el entorno inmediato que permiten suplir un número elevado de necesidades sin la necesidad de utilizar transporte motorizado. Santa Julia, el asentamiento más cercano al centro financiero de la ciudad, se encuentra además contiguo a un eje de servicios metropolitano (Av. Macul), es el que más viajes pedestres exhibe. En contraste los otros asentamientos están inmersos en entornos esencialmente residenciales (Figura 4). Considerando que los cuatro territorios son esencialmente residenciales, con un número acotado de equipamientos en el interior y, en consecuencia, incapaces de resolver todas las necesidades de las mujeres, se esperaría que un número limitado de desplazamientos se mantuviera en el interior del territorio y que la mayoría saliera al resto de la ciudad en búsqueda de oportunidades. Juanita Aguirre y Las Turbinas siguen tal argumento y el 70\% de los viajes se producen hacia el exterior del territorio, mientras que poco más de la mitad de los viajes de Santa Julia y Santo Tomás se producen hacia el exterior (Figura 6).
El bajo porcentaje de desplazamientos de mujeres que se moviliza por el resto de la ciudad y el alto porcentaje de desplazamientos pedestres indica que en estos territorios existen problemáticas que van más allá de la mera localización del asentamiento. Santo Tomás, distante y producto de una política de vivienda reciente, posee índices similares a Santa Julia, cercana a los servicios y con casi cincuenta años de antigüedad.

\section{El espacio público amenazante}

En Santa Julia, los principales focos de atracción de desplazamientos de mujeres de la población corresponden a los equipamientos situados en el interior y en su entorno inmediato. Destaca la presencia de dos escuelas (una interior de primaria y otra exterior de primaria y secundaria), un centro de salud y un hipermercado. Desde estos equipamientos se despliegan grandes redes de movimiento que atraviesan el territorio del asentamiento (Figura 7).

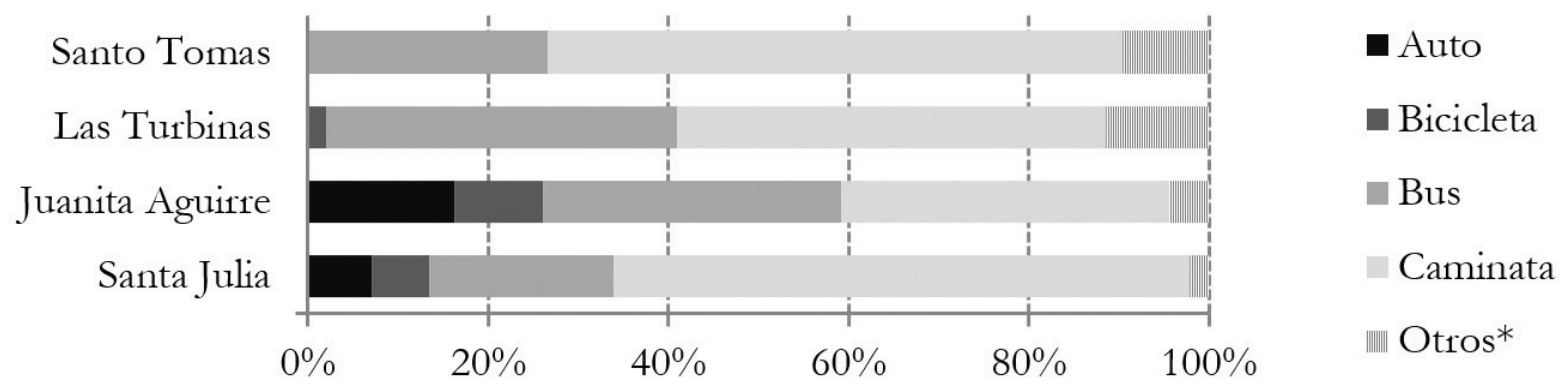

Figura 5 - Porcentaje de viajes que se mantienen en el interior del asentamiento Fuente: SECTRA (2006).

Nota: ${ }^{\star}$ motivos de viaje que representan menos del $3 \%$.

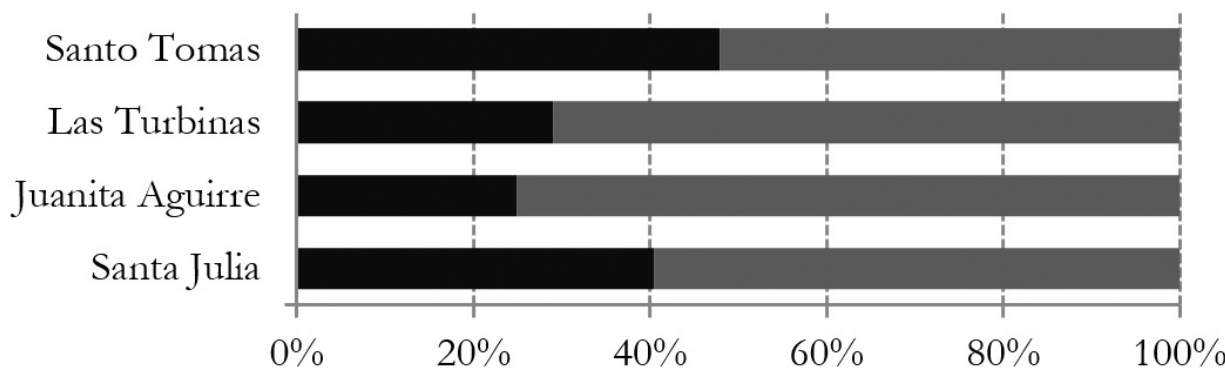

Viajes que se mantienen al interior

Viajes expulsados

Figura 6 - Porcentaje de viajes que se mantienen en el interior o salen del asentamiento Fuente: SECTRA (2006). 


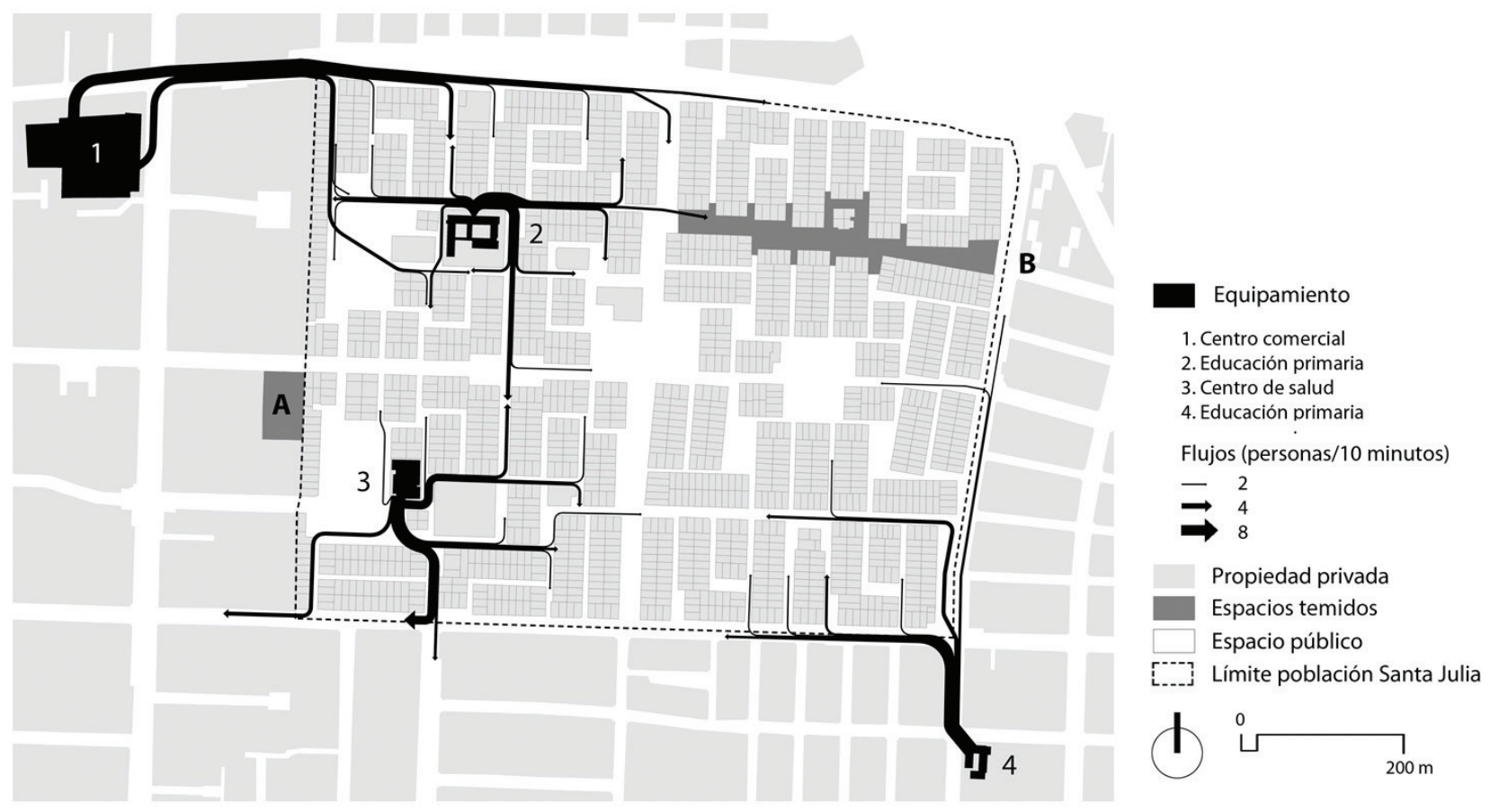

Figura 7 - Redes de movimiento al interior de Santa Julia de Macul Fuente: elaboración propia.

Estas redes revelan que las diferencias observadas en la metrópoli se reproducen a escala barrial. Así, las redes muestran labores de compañía (salud), de provisión (mercado) y la sociabilidad (escuelas). En el centro de salud se observa que la mayoría de las personas que asisten al recinto son mujeres. De las mujeres que ingresan al lugar, un $66 \%$ lo hace en solitario evidenciando que la asistencia al lugar no está necesariamente ligada a sus propias necesidades sino a la de terceros, para solicitar citas médicas o medicamentos.

En el 34\% restante, la mujer actúa como acompañante de terceros, siendo los casos más dramáticos aquellos en los que se desplazan con grupos de niños que no pueden dejarse en casa por temor a la delincuencia. El 13\% de las mujeres asiste acompañando a menores de edad, un $10 \%$ se moviliza con toda la familia (exceptuando alguna figura masculina adulta) y un $11 \%$ acompaña a mayores de edad o a personas con movilidad limitada.

La misma red muestra una constante búsqueda de recorridos temporalmente eficientes. De esta forma, las mujeres describen las rutas más rectas que permite la trama urbana de la población, incluyendo la adición de pequeños pasajes con amplias calles. El diseño y el espacio público pasan a un segundo plano si la ruta provista es directa. La misma eficiencia aparece, aunque con otras características, en la red del centro comercial, en que el camino entre este y las viviendas las mujeres suman una compra en un recinto menor, en el que dos desplazamientos son comprimidos en uno.

Por último, en las escuelas emergen comportamientos sociales donde las mujeres acompañan a los menores al recinto educacional. Mientras esperan que el escolar ingrese al recinto, se encuentran con otras mujeres y entablan conversaciones que se prologan durante los trayectos de regreso a los hogares. El rol de la mujer como soporte social del grupo familiar queda en evidencia.

La suma de los movimientos de mujeres en torno a los equipamientos de la población muestra un uso desequilibrado del territorio. El área nororiente parece no estar integrada a ninguna red de movimiento. Igualmente existe una desconexión en el sector poniente de la población, más específicamente entre las unidades sur-poniente y nor-poniente, causada por un terreno privado ubicado en una vía (Los Espinos) que representa una de las principales salidas de la población (Figura 7). 
En relación con este punto, las mujeres que circulan cotidianamente por la población indican que consideran peligrosas dichas áreas y que por eso no se desplazan por ellas. Ellas mismas indican que la amenaza que se percibe en ambos lugares tiene dos causas: constructos sociales y deterioro del espacio público.

En relación a las construcciones sociales, las mujeres temen al terreno privado (A en Figura 7) por una serie de creencias que lo indican como un lugar abandonado y habitado por antisociales que cometen actos delictuales y abusos contra las mujeres. La calle Los Espinos representa la salida más directa, pero el temor a los rumores hace que las mujeres prefieran realizar un rodeo y utilizar calles distantes. Sacrifican eficiencia por seguridad (Figura 8).

La otra área temida corresponde a un espacio público: la calle Manuel Sánchez (B en Figura 7).
Esta calle representa una amenaza por el deterioro del espacio público, que es interpretado por las mujeres como un riesgo potencial. Presencia de basuras, rayados de muros, zapatos colgados en el tendido eléctrico, entre otros, configuran un espacio amenazante por el cual no se debe circular. Asimismo, las mujeres indican que la presencia de grupos de personas inmóviles también es un signo de peligro (Figura 9).

Frente a los lugares percibidos como peligrosos, las mujeres articulan dos estrategias. La primera, ya nombrada, es evitar las áreas amenazantes, prolongando los recorridos y sacrificando eficiencia y tiempo. La segunda corresponde a la articulación de un sistema de escoltas que consiste en vecinos (normalmente mujeres ancianas) que acompañan a mujeres adolescentes y jóvenes mientras atraviesan un terreno peligroso en el borde de la población.

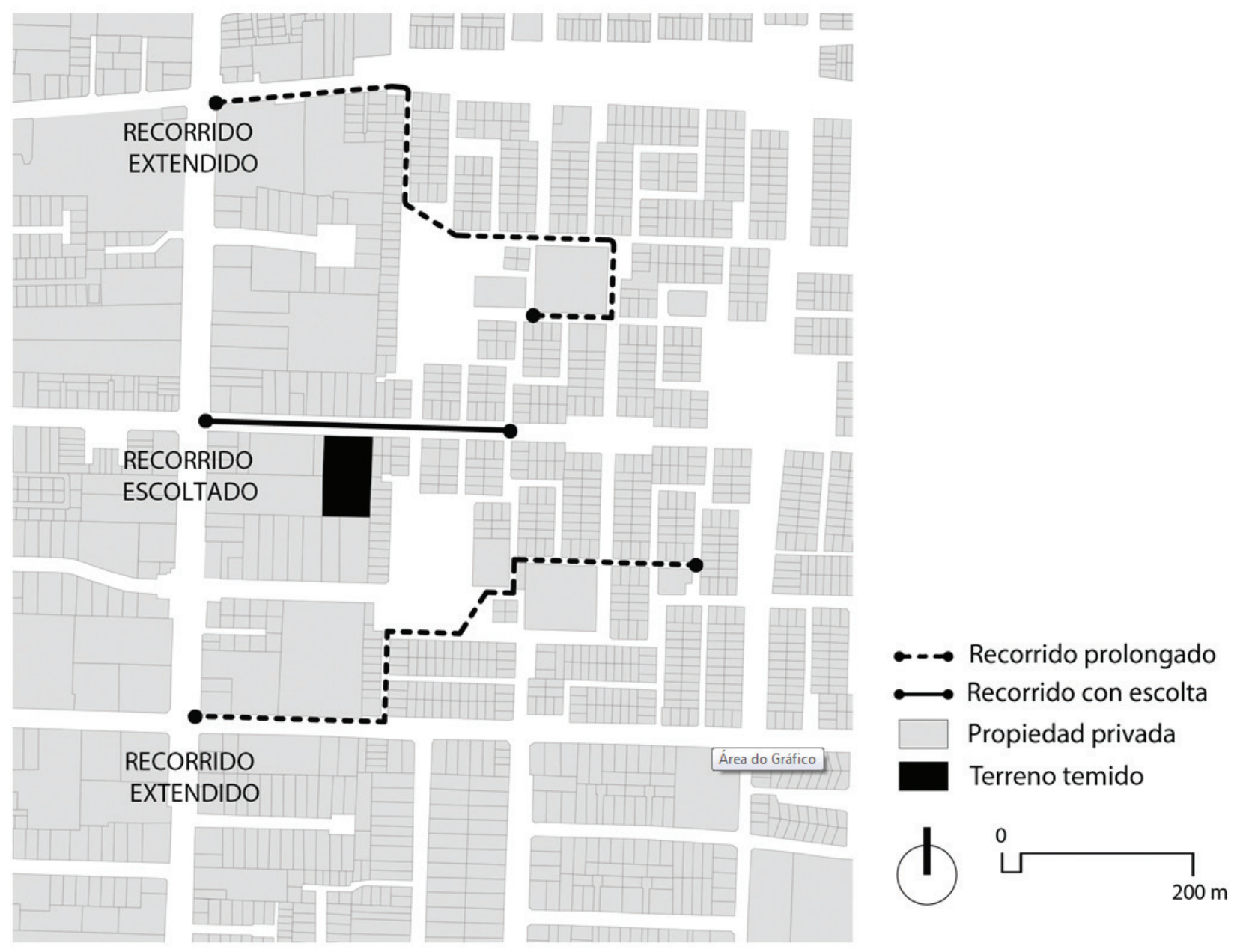

Figura 8 - Estrategias de movilidad frente a la calle Los Espinos en el sector este de Santa Julia Fuente: elaboración propia. 

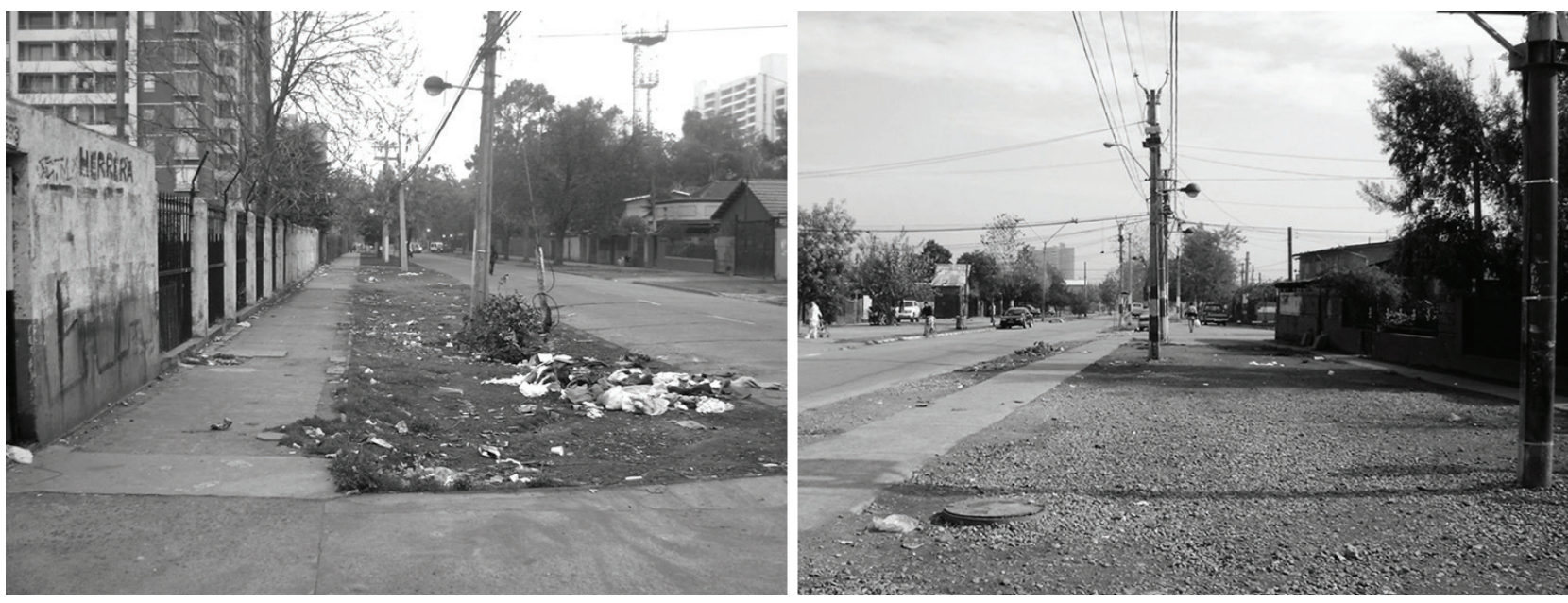

Figura 9 - A la izquierda, veredas frente al recinto privado temido, a la derecha, calle Manuel Sánchez

Fuente: archivo de autores.

Finalmente, una última opción es no realizar el desplazamiento.

El temor en esta población tiende a bloquear las circulaciones e inmovilizar a las mujeres a pesar de que el asentamiento esté bien localizado y su entorno sea relativamente positivo. La inmovilidad de las mujeres deja territorios abandonados, que deben ser evitados, incrementándose la sensación de inseguridad y generándose un círculo vicioso de deterioro.

\section{Conclusiones}

La movilidad de las mujeres en Santiago de Chile muestra que la vulnerabilidad de género tiende a replicarse en las distintas escalas de la ciudad. La construcción social que las asocia con el hogar evidencia limitaciones a escala metropolitana, en donde, en general, se muestran menos móviles que los hombres. De la misma manera, los propósitos de viajes por "compras" son claramente superiores al de los hombres, y el modo más utilizado es la caminata (el modo de más corto alcance), reafirmando la asociación.

Por otro lado, la diferencia de ingresos se ve directamente retratada en la posibilidad de articular más viajes debido al modo de transporte que las mujeres de mayores ingresos prefieren utilizar: el auto. Esto les otorga a los grupos adinerados más facilidades para acceder a servicios y equipamientos que no necesariamente están conectados a los servicios de transporte público.

En el caso de la población Santa Julia, el deterioro del entorno y los rumores de peligro son interpretados por las mujeres como signo de peligro, transformando al espacio público en una amenaza que debe ser evitada, incluso si esto significa no moverse y sacrificar el acceso a las oportunidades que la ciudad ofrece.

\section{Referencias}

Aguirre, R. (1992). Políticas sociales, mujeres y gobierno local (247 p.). Santiago: CIEPLAN.

Alcaíno, P., Domarchi, C., \& López, S. (2009). Gender differences in time use and mobility: time poverty and dual consumption. In Taller Observatorio de Uso del Tiempo. Santiago. Ponencia no publicada.

Alcaíno, P., \& Gutiérrez, P. (2009). Santas o Mundanas: Paradojas y coerciones en el consumo de las mujeres (150 p.). Santiago: Fundación Instituto de la Mujer.

Ascher, F. (2005). Ciudades con velocidad y movilidad múltiples: un desafío para los arquitectos, urbanistas y políticos. Revista ARQ, 60, 11-19.

Banister, D. (1994). Equity and acceptability questions in internalising the social costs of transport. In Organisation for Economic Co-operation and Development \& European Conference of Ministers of Transport (Eds.), Internalising the Social Costs of Transport (p. 153-175). Paris: OCDE. 
Daniels, P. W., \& Warnes, A. M. (1993). Movimiento en ciudades: Transporte y tráfico urbanos (607p.). Madrid: Instituto de Estudios de Administración Local.

Figueroa, C., \& Cortés, A. (2013). Movilidad femenina en asentamientos de escasos recursos en Santiago de Chile: estrategias de movilidad en la búsqueda de eficiencia y seguridad. In Anales de 3er Encuentro Iberoamericano de Movilidad Urbana Sostenible. Lima. Ponencia no publicada.

Gutiérrez, A. (2007). Una aproximación metodológica al estudio de lugares con movilidad vulnerable. In Anales de Congreso Latinoamericano de Transporte Público y Urbano (p. 87-111). Río de Janeiro: PET-COPPE/UFRJ, ANPET. Tema Transporte e Exclusión Social.

Handy, S. (2004). Community design and travel behaviour. In Research on woman's issues in transportation: report of a conference (p. 29-38). Chicago: Transportation Research Board of the National Academies.

Hayden, D. (1981). The grand domestic revolution: a history of feminist designs for American homes, neighborhoods, and cities. (13a ed., 367 p.). Cambridge: The MIT Press.

Hercé, M. (2009). Sobre la movilidad en la ciudad: propuestas para recuperar un derecho ciudadano (321 p.). Barcelona: Editorial Reverté. Estudios universitarios de arquitectura, 8.

Hidalgo, R. (2005). La vivienda social en Chile y la construcción del espacio urbano en el Santiago del siglo XX. (491 p.). Santiago: Instituto de Geografía PUC/Centro de investigaciones Barros Arana.

HOGAR de Cristo. (1973). 52\% del mundo sin casa (52 p.). Santiago: SELAVIP.

Izard, M. (1985). Marginados, fronterizos, rebeldes y oprimidos. (199 p.). Madrid: Serbal.

Jirón, P. (2007). Implicancias de Género en las experiencias de movilidad cotidiana urbana en Santiago de Chile. Revista Venezolana de Estudios de la Mujer, 12(29), 173-197.

Kaufmann, V. (2001). La motilité: une notion clé pour revisiter l'urbain. In M. Bassand, V. Kaufmann, \& D. Joya (Eds.), Enjeux de la sociologie urbaine (p. 87-102). Lausanne: Presses polytechnique et universitaire romandes.

Law, R. (1996). Beyond "women and transport": towards new geographies of gender mobility. Progress in Human Geography, 23(4), 567-588.

Loukaitou-Sideris, A. (2004). Is It Safe to Walk Here? Design and Policy Responses to Women's Fear of
Victimization in Public Places. In Research on woman's issues in transportation: report of a conference (p. 102-112). Chicago: Transportation Research Board of the National Academies.

Markovich, J., \& Hendler, S. (2006). Beyond "Soccer Moms": Feminism and New Urbanism critical approaches to suburbs. Journal of Planning Education and Research, 25(4), 410-427.

McFadden, D. (1974). The Measurement of urban travel demand. Journal of Public Economics, 3, 303-328.

Módenes, J. A. (2007). Movilidad espacial: uso temporal de territorio y poblaciones vinculadas. In Anales de In X Congreso de la Población Española. Navarra. Ponencia no publicada.

Ortúzar, J. D., \& Willumsen, L. Modelling Transport. Cuarta ed. Chichester: John Wiley and Sons, 2011.

Perrot, M. (1997). Mujeres en la ciudad (159 p.). Santiago: Andrés Bello.

Perulli, P. (1995). Atlas Metropolitano (134 p.). Madrid: Alianza.

Primerano, F., Taylor, M., Prtaksringkarn, L., \& Tisato, P. (2008). Defining and understanding trip chaining behavior. Transportation Research Record, 35(1), 55-72.

Rainero, L. (2006). Herramientas para la promoción de ciudades seguras desde la perspectiva del género (86 p.). Córdoba: CISCSA.

Rainero, L. (2001). Una Mirada de género a los asentamientos humanos. Medio Ambiente y Urbanización: Gestión Urbana, Género y Programas Sociales, 17(56), 73-92.

Rodigou, M. (2009). Territorios prohibidos: la violencia hacia las mujeres en la ciudad. In Anales de VI Encuentro Interdisciplinario de Ciencias Sociales y Humanas. Córdoba. Ponencia no publicada.

Rosenbloom, S. (2004). Understanding Women's and Men's Travels Patters. In Research on woman's issues in transportation: report of a conference. Chicago: Transportation Research Board of the National Academies.

Sabatini, F. (2000). Reforma de los mercados de suelo en Santiago, Chile: efectos sobre los precios de la tierra y la segregación residencial. EURE. Revista Latinoamericana de Estudios Urbano Regionales, 26(77), 49-80.

Secretaría de Planificación y Transporte - SECTRA. (1991). Encuesta Origen Destino 1991. Santiago. 
Secretaría de Planificación y Transporte - SECTRA. (2006). Encuesta Origen Destino 2006. Santiago.

Sugranyes, A. (2005). La política habitacional en Chile, 1980-2000: un éxito liberal para dar techo a los pobres. In A. Rodríguez \& A. Sugranyes (Eds.), Los con techo. Un desafío para la política de vivienda social (p. 23-58). Santiago: Sur Ediciones.

Thieme, C. (2010). Elección de establecimientos educacionales en Chile - ¿Por qué no ha existido presión por calidad? Santiago: Universidad Diego Portales. Documentos de Trabajo, 2.

Tokman, A. (2006). El Minvu, la política habitacional y la expansión excesiva de Santiago. In A. Galetovic (Ed.),
Santiago. Dónde estamos y hacia dónde vamos (p. 489-521). Santiago: Centro de Estudios Públicos.

Valcárcel, A., \& Quiroz, B. 2008. La política de las mujeres (4a ed., 233 p.). Madrid: Cátedra.

Valenzuela, M. E. (ed.), (2003). Mujeres, pobreza y mercado de trabajo. Argentina y Paraguay (160 p.). Santiago: Oficina regional de la OIT para América Latina y el Caribe.

Valiquette, F., \& Morency, C. (2010). Trip chaining and its impact on travel behaviour. In Anales de $12^{\circ}$ WCTR. Lisboa. Ponencia no publicada.

Recibido: Abr. 16, 2014

Aprobado: Agosto 30, 2014 